\title{
OPTIMAL LIPSCHITZ EXTENSIONS AND THE INFINITY LAPLACIAN
}

M. G. Crandall (1), Department of Mathematics, UC Santa Barbara

L. C. Evans ${ }^{(2)}$, Department of Mathematics, UC Berkeley

R. F. Gariepy, Department of Mathematics, University of Kentucky

\begin{abstract}
We reconsider in this paper boundary value problems for the so-called "infinity Laplacian" PDE and the relationships with optimal Lipschitz extensions of the boundary data. We provide some fairly elegant new proofs, which clarify and simplify previous work, and in particular draw attention to the fact that solutions may be characterized by a comparison principle with appropriate cones. We in particular show how comparison with cones directly implies the variational principle associated with the equation. In addition, we establish a Liouville theorem for subsolutions bounded above by planes.
\end{abstract}

\section{INTRODUCTION}

A typical problem in the conventional calculus of variations asks us to find a function which minimizes an integral expression of the form, say,

$$
I[u]:=\int_{U} F(D u) d x,
$$

subject to given boundary conditions, where $U \subset R^{n}$ is open, $u: U \rightarrow R$, and $D u=$ $\left(u_{x_{1}}, \ldots, u_{x_{n}}\right)$ is the gradient. Here $F: R^{n} \rightarrow R$ is a given nonlinearity: $F(D u)$ can of course often be interpreted as some sort of energy density.

The past decade has seen great interest in some highly nonconventional variants, which amount to "calculus of variations problems in the sup-norm": see for instance the survey by Barron [4] and the references cited there. The simplest such model problem in effect replaces the integral energy (1.1) by the pointwise functional

$$
I[u]:=\|D u\|_{L^{\infty}(U)} .
$$

\footnotetext{
${ }^{(1)}$ Supported in part by a Miller Research Professorship, UC Berkeley, 1996.
}

${ }^{(2)}$ Supported by NSF Grant DMS-942342 
A central question has been to understand minimization problems involving this and related functionals, and especially to develop appropriate PDE methods.

Now problem (1.1) leads as usual to the Euler-Lagrange equation. A central discovery of G. Aronsson [1], [2] was that likewise problem (1.2), properly interpreted, entails a highly nonlinear and highly degenerate PDE. This is the equation

$$
-\Delta_{\infty} u=0 .
$$

In this formula $\Delta_{\infty}$ denotes the so-called "infinity-Laplacian", defined on smooth real-valued functions $u=u(x)$ by

$$
\Delta_{\infty} u:=\sum_{j, k=1}^{n} u_{x_{j}} u_{x_{k}} u_{x_{j} x_{k}} .
$$

It remains a major challenge to elucidate fully the connections between analytic properties of solutions to boundary-value problems for (1.3), (1.4) and the variational problem associated with (1.2).

Derivation. The name arises this way. Consider first for finite $p$ the nonlinear $p$ Laplacian operator:

$$
\Delta_{p} u=\operatorname{div}\left(|D u|^{p-2} D u\right)=(p-2)|D u|^{p-4} \Delta_{\infty} u+|D u|^{p-2} \Delta u
$$

The equation $-\Delta_{p} u=0$ arises of course as the Euler-Lagrange equation for the problem of minimizing

$$
I_{p}[u]:=\frac{1}{p} \int_{U}|D u|^{p} d x
$$

subject to given boundary conditions.

Now our formally dividing the equation $-\Delta_{p} u=0$ by $(p-2)|D u|^{p-4}$ leads to the equation

$$
-\Delta_{\infty} u-\frac{1}{p-2}|D u|^{2} \Delta u=0 .
$$

(Note that the division by $|D u|^{p-4}$ is of no consequence even on the set where $D u=0$, for the resulting expression also vanishes when $D u=0$.)

Standard theorems about viscosity solutions (see for example [7]) imply that if $u_{p}$ solves $-\Delta_{\infty} u_{p}-\frac{1}{p-2}\left|D u_{p}\right|^{2} \Delta u_{p}=0$ and $u_{p} \rightarrow u$ locally uniformly along a sequence $p \rightarrow \infty$, then $-\Delta_{\infty} u=0$ in the viscosity sense. 
Absolutely minimizing Lipschitz extensions. R. Jensen [10], stimulated by the work of Aronsson, proved the first basic theorems about solvability of the the Dirichlet problem

$$
-\Delta_{\infty} u=0 \quad \text { in } \quad U, \quad u=g \quad \text { on } \quad \partial U .
$$

Jensen proved ([10], Section 3) that if $U$ is a bounded, open, connected subset of $\mathbb{R}^{n}$ and $g \in C(\partial U)$, then (DP) has a unique viscosity solution $u \in C(\bar{U})$ satisfying $u=g$ on $\partial U$.

Jensen showed as well that this solution is also characterized by following variational property, first identified by Aronsson: for every open bounded subset $V$ of $U$ and for each $v \in C(\bar{V})$, one has

$$
u=v \quad \text { on } \quad \partial V \quad \text { implies } \quad\|D u\|_{L^{\infty}(V)} \leq\|D v\|_{L^{\infty}(V)} .
$$

We say that $u$ is an "absolutely minimizing Lipschitz extension" (abbreviated AML) of $g$.

(We adopt the convention that $\|D v\|_{L^{\infty}(U)}=\infty$ if $D v$, taken in the sense of distributions, does not belong to $L^{\infty}(U)$. We will also write " $u$ is AML" to mean that the AML property holds, whether or not we are thinking of $u$ as an extension of any kind.)

Outline of this paper. We offer several contributions to this circle of problems.

In Section 2 we present some preliminaries and definitions, primarily the notion of "comparison with cones". Then we prove that solutions of $-\Delta_{\infty} u \leq 0$ enjoy comparison with cones from above. We use this simple property to derive continuity properties of subsolutions and some further consequences. These preliminary results are related to various observations of Jensen [10], Section 3, but we have made our presentation completely self-contained.

In Section 3 we establish the equivalence of three properties: $-\Delta_{\infty} u=0$, comparison with cones, and the AML property. The derivation of the equation $-\Delta_{\infty} u=0$ in the viscosity sense from the AML property is due to Jensen, but our methods are simpler and more useful. (Aronsson had earlier shown this for smooth u.) Our proof is intrinsic, using only comparison with cones, and makes no reference to the $p$-Laplacian, as in [10], [5].

A corollary is that any everywhere differentiable solution $v$ of an eikonal equation $|D v|=$ constant is AML, and so "infinity harmonic".

In Section 4 we prove that a subsolution in $\mathbb{R}^{n}$ which lies below a plane is linear; that is, if $-\Delta_{\infty} u \leq 0$ and $u(x) \leq a+\langle p, x\rangle$ for fixed $a \in \mathbb{R}, p \in \mathbb{R}^{n}$ and all $x \in \mathbb{R}^{n}$, then 
$u=u(0)+\langle p, x\rangle$. The simple special case in which $p=0$ is a Liouville type theorem stating that global subsolutions of $-\Delta_{\infty} u=0$ which are bounded above are constant.

This modest result provides the only positive evidence we know towards the conjecture that a global Lipschitz continuous solution must be linear. This stronger result, if true, would be positive evidence toward a major unsolved problem for $-\Delta_{\infty}$ : Are solutions of $-\Delta_{\infty} u=0$ continuously differentiable?

As a small step in this direction we show as well that the PDE $-\Delta_{\infty} u \leq 0$ implies that if $u-\varphi$ has a maximum at some point $\hat{x}$ at which $\varphi$ has a derivative $D \varphi(\hat{x})$, then so does $u$ and $D u(\hat{x})=D \varphi(\hat{x})$.

Other work. Generalizations of the associated variational problem are considered in Juutinen [11] and $\mathrm{Wu}$ [15]. In [11] one finds variants of a number of our results, with heavier machinery to deal with the more general equations. Primary differences include that we do not use approximation by problems in $L^{p}$ and we show that the very explicit property of comparison with cones suffices to replace the quite implicit property called " $(F, \infty)$-subharmonic" in [11]. In addition, the significance of the equation $-\Delta_{\infty} u$ for image processing is brought out in Caselles, Morel and Sbert [6], wherein the regularity question is also of some interest.

We note that we have not proved uniqueness in this framework. At present this has only been accomplished using $-\Delta_{p}$ and obstacle problems in Jensen's original work, which was generalized as regards the equation in Juutinen [11] and $\mathrm{Wu}[15]$.

Notation. $U$ will always be an open subset of $\mathbb{R}^{n}$. For any set $V \subset \mathbb{R}^{n}, \partial V$ is its boundary and $\bar{V}$ is its closure. The notation

$$
V \subset \subset U
$$

means that $V$ is an open subset of $U$ whose closure $\bar{V}$ is a compact subset of $U$. In addition

$$
B_{r}\left(x_{0}\right)=\left\{y \in \mathbb{R}^{n} ;\left|y-x_{0}\right|<r\right\}, B_{r}=B_{r}(0) \quad \text { and } \quad S_{r}\left(x_{0}\right)=\partial B_{r}\left(x_{0}\right) .
$$

Here $|x|$ is the Euclidean length of $x$. Below $\langle x, y\rangle=\sum_{i=1}^{n} x_{i} y_{i}$ denotes the Euclidean inner-product.

For any subset $V \subset \mathbb{R}^{n}$, we put

$$
\begin{aligned}
& \operatorname{USC}(V)=\{u: V \mapsto \mathbb{R} ; u \text { is upper semicontinuous }\}, \\
& \operatorname{LSC}(V)=\{u: V \mapsto \mathbb{R} ; u \text { is lower semicontinuous }\} .
\end{aligned}
$$




\section{Preliminaries}

In this section we introduce the idea of comparison with cones, and derive some simple continuity and other properties of functions satisfying this condition. In Section 3 we prove that comparison with cones implies $-\Delta_{\infty}=0$, and once this is known some of the following assertions are close to those available in the literature: see Jensen [10], Juutinen [11], Lindqvist-Manfredi [12], [13], etc. A main point is that our self-contained presentation is straightforward and nowhere invokes approximation by the $p$-Laplacian.

We begin with definitions:

Definition 2.1 (i) A function $u: U \rightarrow \mathbb{R}$ is a viscosity solution of $-\Delta_{\infty} u \leq 0$ in $U$ if $u \in \operatorname{USC}(U)$ and for every local maximum point $\hat{x} \in U$ of $u-\varphi$, where $\varphi$ is $C^{2}$ in some neighborhood of $\hat{x}$, we have $-\Delta_{\infty} \varphi(\hat{x}) \leq 0$. Furthermore the expressions " $u$ is a viscosity solution of $-\Delta_{\infty} u \leq 0$ " and " $u$ is a viscosity subsolution of $-\Delta_{\infty} u=0$ " have identical meanings.

(ii) A function $u: U \rightarrow \mathbb{R}$ is a viscosity solution of $-\Delta_{\infty} u \geq 0$ in $U$ if $u \in \operatorname{LSC}(U)$ and for every local minimum point $\hat{x} \in U$ of $u-\varphi$, where $\varphi$ is $C^{2}$ in some neighborhood of $\hat{x}$, we have $-\Delta_{\infty} \varphi(\hat{x}) \geq 0$.

(iii) Moreover, $u$ is a viscosity solution of $-\Delta_{\infty} u=0$ in $U$ if it is both a viscosity subsolution and a viscosity supersolution in $U$.

In this paper the unmodified terms subsolution, supersolution and solution are always understood in the viscosity sense. Since the formal expression $-\Delta_{\infty} u$ is odd in $u, u$ is a subsolution of $-\Delta_{\infty} u=0$ in $U$ if and only if $v=-u$ is a supersolution. Thus every result for a subsolution implies an analogous result for supersolutions, and conversely.

We next introduce the property of "comparison with cones". First, notice that any solution of an eikonal equation $|D v|=$ constant is a solution of $-\Delta_{\infty} v=0$ wherever it is smooth. Thus $v(x)=a+b\left|x-x_{0}\right|$ is a solution for $x \neq x_{0}$. If $b$ is positive, then $v$ is a global subsolution, but it is not a global solution. Comparison with these particular solutions is the basic tool of the theory. 
Lemma 2.2 Let $U$ be bounded, $u \in \operatorname{USC}(\bar{U})$ and $-\Delta_{\infty} u \leq 0$ in $\Omega$. If $x_{0} \in \mathbb{R}^{n}$ and $a, b \in \mathbb{R}$, and

$$
u(x) \leq C(x)=a+b\left|x-x_{0}\right| \quad \text { for } \quad x \in \partial\left(U \backslash\left\{x_{0}\right\}\right)
$$

then

$$
u(x) \leq C(x) \text { for } \quad x \in U
$$

Proof. Assume that $0<u(\hat{x})-C(\hat{x})$ at some point $\hat{x} \in U \backslash\left\{x_{0}\right\}$. Choose $R$ so large that $\left|x-x_{0}\right| \leq R$ on $\partial U$ and put $w=a+b\left|x-x_{0}\right|+\epsilon\left(R^{2}-\left|x-x_{0}\right|^{2}\right)$. Then $u \leq w$ on $\partial\left(U \backslash\left\{x_{0}\right\}\right)$, whereas $u(\hat{x})-w(\hat{x})>0$ if $\epsilon$ is sufficiently small. We may assume that $\hat{x}$ is the maximum of $u-w$ on $U \backslash\left\{x_{0}\right\}$. A direct computation shows that $-\Delta_{\infty} w(\hat{x})=2 \epsilon\left(2 \epsilon\left|\hat{x}-x_{0}\right|-b\right)^{2}$, and this is strictly positive if either $b \leq 0$ or if $b>0$ and $\epsilon$ is sufficiently small. This contradicts the assumption $-\Delta_{\infty} u \leq 0$.

We give the comparison property of Lemma 2.2 a name:

Definition 2.3 Let $u: U \mapsto \mathbb{R}$. Then $u$ enjoys comparison with cones from above in $U$ if for every bounded open subset $V$ of $U$ and every $x_{0} \in \mathbb{R}^{n}, a, b \in \mathbb{R}$ for which

$$
u(x) \leq C(x)=a+b\left|x-x_{0}\right|
$$

holds on $\partial\left(V \backslash\left\{x_{0}\right\}\right)$, we have $u \leq C$ in $V$ as well.

Comparison with cones from below has a similar definition. Moreover, u enjoys comparison with cones in $U$ if it enjoys comparison with cones both from above and below.

Solutions of $-\Delta_{\infty} u \geq 0$ in $U$ enjoy comparison with cones from above, and likewise supersolutions enjoy comparison with cones from below. Thus solutions of $-\Delta_{\infty} u=0$ enjoy comparison with cones. In the next section we prove that the property of comparison with cones is in fact equivalent to $-\Delta_{\infty} u=0$.

Lemma 2.4 Let $u \in \mathrm{USC}(U)$ enjoy comparison with cones from above. Then the function defined for $y \in U$ and $r<\operatorname{dist}(y, \partial U)$ by

$$
L_{r}(y):=\max _{z \in S_{r}(y)} \frac{u(z)-u(y)}{r}
$$


is nondecreasing in $r$ and nonnegative.

Proof. $L_{r}(y)$ is the smallest constant for which

$$
u(x) \leq u(y)+L_{r}(y)|x-y|
$$

holds for $|x-y|=r$. Comparison with cones then implies the inequality holds for $|x-y| \leq r$. Thus $(u(x)-u(y)) /|x-y| \leq L_{r}(y)$ for $|x-y| \leq r$, and this establishes the monotonicity.

In order to show that $0 \leq L_{r}(y)$, it suffices to take $y=0$ and show that $0 \leq \lim _{r \downarrow 0} L_{r}(0)$. Let

$$
M>\lim _{r \downarrow 0} L_{r}(0) ;
$$

so that the above inequality implies

$$
u(x) \leq u(0)+M|x| \text { for } \quad|x|<r_{0}
$$

provided $L_{r}(0)<M$ for $r<r_{0}$. Now we also have

$$
u(0) \leq u(x)+\max _{z \in S_{r}(x)} \frac{u(z)-u(x)}{r}|x| \text { for }
$$

for $r>|x|$ and sufficiently small. Use (2.6) with $x=z$ and $|z| \leq|x|+r$ in (2.7) and conclude that

$$
u(0) \leq u(x)+\frac{u(0)+M(|x|+r)-u(x)}{r}|x| .
$$

Then

$$
-M \frac{r+|x|}{r-|x|}|x| \leq u(x)-u(0)
$$

and so

$$
-M \frac{r+\epsilon}{r-\epsilon} \leq \max _{|x|=\epsilon} \frac{u(x)-u(0)}{\epsilon} .
$$

Let $\epsilon \downarrow 0$ to conclude that if $(2.5)$ holds, then $-M \leq \lim _{r \downarrow 0} L_{r}(0)$. This in turn implies $\lim _{r \downarrow 0} L_{r}(0) \geq 0$.

We turn to continuity properties which may be deduced from comparison with cones. 
Lemma 2.5 Let $u \in \operatorname{USC}(U)$ enjoy comparison with cones from above. Then $u \in W_{\text {loc }}^{1, \infty}(U)$, and for almost all $x$

$$
|D u(x)| \leq \max _{z \in S_{r}(x)} \frac{u(z)-u(x)}{r} \quad \text { for } r<\operatorname{dist}(x, \partial U) .
$$

Proof. As above, we have

$$
u(x) \leq u(y)+\max _{z \in S_{r}(y)} \frac{u(z)-u(y)}{r}|x-y| \quad \text { for } \quad|x-y| \leq r \quad \text { and } \quad r<\operatorname{dist}(y, \partial U),
$$

since the inequality holds for $|x-y|=r$. Note next that $u$ is bounded below on compact subsets of $U$. Indeed, if $|x-y|<r$ in (2.9), we find

$$
\left(\frac{r}{r-|x-y|}\right) u(x)-\max _{z \in S_{r}(y)} u(z) \frac{|x-y|}{r-|x-y|} \leq u(y) .
$$

For each $r$ the expression on the left is lower semicontinuous in $y \in B_{r}(x)$ and $r<$ dist $(y, \partial U)$. Consequently $u(y)$ is locally bounded below.

Let $L_{r}$ be given by (2.4). Using the upper semicontinuity of $u$ and the local boundedness below just proved, $L_{r}(y)$ is locally bounded below and above for fixed $r$. By Lemma $2.4 L_{r}$ is nonnegative and decreases as $r$ decreases. So we can define

$$
L(y)=\lim _{r \downarrow 0} L_{r}(y) .
$$

Note that if $u$ happens to be differentiable at $y$, then from the definitions we find

$$
L(y)=|D u(y)|
$$

We now know that $0 \leq L_{r}(y)$ is bounded above for fixed $r$ and $y$ in a compact subset of dist $(y, \partial U)<r$ and decreases as $r$ decreases. Interchanging $x$ and $y$ in (2.9) and putting the resulting relations together yields

$$
|u(x)-u(y)| \leq \max \left(L_{r}(y), L_{r}(x)\right)|x-y|
$$

for $|x-y| \leq r$ and $\max (\operatorname{dist}(x, \partial U)$, dist $(y, \partial U)<r$. We conclude that $u$ is locally Lipschitz continuous. Then $L_{r}(y)$ is continuous in $y$ and $L(y)$ is upper semicontinuous, by the monotone nature of the defining limit. We conclude that $u$ is differentiable a.e. and for a.e. $x$

$$
|D u(x)| \leq L(x) \leq L_{r}(x) \quad \text { for } \quad \operatorname{dist}(x, \partial U)<r
$$


We derive some consequences of Lemmas 2.2 and 2.5:

Corollary 2.6 (i) Let $u$ enjoy comparison with cones from above in $\mathbb{R}^{n}$. If $p \in \mathbb{R}^{n}$, a $\in \mathbb{R}$ and $u(x) \leq a+\langle p, x\rangle$ for $x \in \mathbb{R}^{n}$, then $|D u| \leq|p|$ a.e.

(ii) If $u$ enjoys comparison with cones from above in $\mathbb{R}^{n}$, then

$$
|D u(x)| \leq \frac{2\|u\|_{L^{\infty}\left(B_{r}(x)\right)}}{r} \quad \text { for } \quad r<\operatorname{dist}(x, \partial U)
$$

a.e. in $U$.

Proof. By Lemma 2.5 and the assumptions, the inequalities

$$
|D u(x)| \leq \max _{|z-x|=r} \frac{u(z)-u(x)}{r} \leq \max _{|z-x|=r} \frac{a+\langle p, z\rangle-u(x)}{r} \leq \frac{|a|+|p|(|x|+r)-u(x)}{r}
$$

hold a.e. Let $r \rightarrow \infty$ to prove the first assertion. The second statement follows immediately from Lemma 2.5.

If $u$ enjoys comparison with cones from above and below in $U$, let us define

$$
L_{r}^{+}(y)=\max _{z \in S_{r}(y)} \frac{u(z)-u(y)}{r} \text { and } L_{r}^{-}(y)=\min _{z \in S_{r}(y)} \frac{u(z)-u(y)}{r}
$$

for $r<\operatorname{dist}(y, \partial U)$, as well as the limits

$$
L^{+}(y)=\lim _{r \downarrow 0} L_{r}^{+}(y) \quad \text { and } \quad L^{-}(y)=\lim _{r \downarrow 0} L_{r}^{-}(y)
$$

As above, $L_{r}^{+}(y)$ increases as $r \downarrow 0$ and $L_{r}^{-}(y)$ decreases. We have $L_{r}^{+}(y) \geq 0$ and $L_{r}^{-}(y) \leq 0$ by Lemma 2.4. By Lemma $2.5 u$ is differentiable a.e., and at a point $x$ of differentiability (2.12) implies $L^{+}(x)=|D u(x)|, L^{-}(x)=-|D u(x)|$ a.e., and so $L^{+}(x)=-L^{-}(x)$ a.e. In fact, this holds everywhere:

Lemma 2.7 Let $u \in C(\bar{U})$ enjoy comparison with cones in $\bar{U}$. Then

(i) $L^{+}(y)=-L^{-}(y)$ for $y \in U$. 
(ii) $\sup _{y \in U} L^{+}(y)=\|D u\|_{L^{\infty}(U)}$.

Proof. Put $y=0$ and let $M>L^{+}(0)$. Then (2.8) holds as before, and so

$$
-M \frac{r+\epsilon}{r-\epsilon} \leq \min _{|x|=\epsilon} \frac{u(x)-u(0)}{\epsilon} .
$$

Sending $\epsilon \downarrow 0$, it follows that $-M \leq V_{-}(0)$ and then that $-V_{+}(0) \leq V_{-}(0)$. The other inequality is proved the same way (or replace $u$ by $-u$.)

We now prove (ii). The inequality $L^{+}(x) \leq\|D u\|_{L^{\infty}(U)}$ follows from (2.15). On the other hand, we may choose Lebesgue points $x_{j} \in U$ of $D u$ (in the sense of $L^{n}$ ) where $u$ is differentiable so that $\left|D u\left(x_{j}\right)\right| \geq\|D u\|_{L^{\infty}(U)}-1 / j$. Then $L^{+}\left(x_{j}\right)=\left|D u\left(x_{j}\right)\right|$ by $(2.12)$ shows that (ii) holds.

\section{Equivalences}

The next result contains the implications

$$
A M L \quad \Longrightarrow \quad-\Delta_{\infty} u=0 \quad \Longleftrightarrow \quad \mathrm{u} \text { enjoys comparison with cones. }
$$

The full equivalence of the three properties is established later.

Theorem 3.1 Let $u \in C(U)$. If $u$ satisfies the $A M L$ property in $U$, then $-\Delta_{\infty} u=0$ in $U$. Moreover, $-\Delta_{\infty} u=0$ in $U$ if and only if $u$ enjoys comparison with cones in $U$.

Proof. We already know that $-\Delta_{\infty} u \leq 0$ implies that $u$ enjoys comparison with cones from above, etc., via Lemma 2.2.

We turn to the derivation of the equation $-\Delta_{\infty} u=0$ from both the AML property and from comparison with cones. Suppose $u$ is defined in some open set and fails to solve $-\Delta_{\infty} u=0$. We will show first that then there is an bounded, connected, open set $V$ within the domain of $u$ such that $\|D u\|_{L^{\infty}(V)}$ exceeds the (finite) Lipschitz constant $L$ of $\left.u\right|_{\partial V}$ :

$$
L=\left\{\frac{|u(x)-u(y)|}{|x-y|}: x, y \in \partial V, x \neq y\right\} .
$$

This contradicts the AML property, for there is always a Lipschitz continuous extension $v$ of $\left.u\right|_{\partial V}$ to $\mathbb{R}^{n}$ which has the same Lipschitz constant, and then $u=v$ on $\partial V$, whereas 
$\|D v\|_{L^{\infty}(V)} \leq L<\|D u\|_{L^{\infty}(V)}$. Elements of the same construction will show also that then comparison with cones fails.

Since $u$ and $-u$ solve both solve $-\Delta_{\infty} w=0$ if either does, we assume that $u$ is not a subsolution at 0 . Adding a constant to $u$, we may also assume $u(0)=0$. This means that there exists $\varphi \in C^{2}$ such that

$$
u(x) \leq \varphi(x)-\varphi(0) \quad \text { near } \quad 0 \quad \text { and } \quad-\varphi_{x_{i}}(0) \varphi_{x_{j}}(0) \varphi_{x_{i}, x_{j}}(0)>0 .
$$

We may rotate and reflect coordinates to assume that $D \varphi(0)=(p, 0, \ldots, 0)$ where $p>0$. Noting that (3.1) then entails $\varphi_{x_{1}, x_{1}}(0)=a<0$, we may rescale and assume $a=-4$. Writing $x=x_{1} e_{1}+y$ where $e_{1}$ is the unit vector in the first coordinate direction and $y$ is perpendicular to it, we have

$$
\left\langle D^{2} \varphi(0)\left(x_{1} e_{1}+y\right), x_{1} e_{1}+y\right\rangle \leq-4 x_{1}^{2}+K\left(\left|x_{1}\right||y|+|y|^{2}\right) \leq-3 x_{1}^{2}+K|y|^{2}
$$

with various $K$ and strict inequality in a deleted neighborhood of the origin.

Writing hereafter $x$ in place of $x_{1}$, we use the first inequality of (3.1), (3.2) and the continuity of $D^{2} \varphi$ to deduce that for a suitable new constant $K$

$$
\begin{aligned}
u(x, y) & =u\left(x e_{1}+y\right) \leq \varphi\left(x e_{1}+y\right)-\varphi(0) \\
& =\left\langle D \varphi(0), x e_{1}+y\right\rangle+\frac{1}{2}\left\langle D^{2} \varphi(0)\left(x e_{1}+y\right), x e_{1}+y\right\rangle+\mathrm{o}\left(x^{2}+|y|^{2}\right) \\
& \leq p x-x^{2}+K|y|^{2}
\end{aligned}
$$

in a neighborhood of $(x, y)=(0,0)$, with strict inequality except at the origin. We are writing $(x, y)$ and $x e_{1}+y$ interchangeably.

To continue, we show below that for small $r$ the cone

$$
C(x, y)=-p r-r^{2}+p|(-r, 0)-(x, y)|
$$

which has $p$ as a Lipschitz constant, satisfies

$$
u(x, y)<p x-x^{2}+K|y|^{2} \leq C(x, y) \quad \text { on } \quad \partial B_{r}
$$

where $B_{r}$ is the ball of radius $r$ centered at the origin. Since $C(0,0)<0=u(0,0),(3.4)$ implies that there is a connected open neighborhood of $V$ of 0 with $u=C$ on $\partial V$ and $u<C$ in $V$. Thus $p$ is a Lipschitz constant for $\left.u\right|_{\partial V}=\left.C\right|_{\partial V}$. However, $u(x, 0)-u(0,0)=$ 
$u(x, 0) \leq p x-x^{2}$ for $-r \leq x<0$ implies $|u(x, 0)-u(0,0)| /|x| \geq p+|x|>p$ for such $x$. This completes the argument: if $u$ is not a solution of $-\Delta_{\infty} u=0$, then $u$ does not satisfy the AML condition. Moreover, since $u \leq C$ on $\partial B_{r} \ni(-r, 0)$ while $C(0,0)<u(0,0), u$ does not enjoy comparison with cones. Thus comparison with cones and AML both imply $-\Delta_{\infty} u=0$.

It remains to check that $p x-x^{2}+K|y|^{2} \leq C$ on $\partial B_{r}$ if $r$ is small. This amounts to

$$
p x-x^{2}+K|y|^{2} \leq-p r-r^{2}+p\left((x+r)^{2}+|y|^{2}\right)^{1 / 2} \quad \text { for } \quad x^{2}+|y|^{2}=r^{2} .
$$

First note that if $x=-r$ or $x=r$ (so $y=0$ ), then (3.5) holds with equality. Using $|y|^{2}=r^{2}-x^{2}$ results in the equivalent formulation

$$
p x-(K+1) x^{2}-p \sqrt{2 r}(r+x)^{1 / 2} \leq-p r-r^{2}-K r^{2} \quad \text { for } \quad-r \leq x \leq r .
$$

The left hand side is convex in $x \in[-r, r]$ for small $r$ (the second derivative of $-\sqrt{2 r}(r+x)^{1 / 2}$ is bounded below by $C / r$ ). Hence the inequality holds if and only if it holds at the end points, and this was already verified.

The proof that comparison with cones implies $-\Delta_{\infty} u=0$ is one-sided: comparison with cones from above implies $-\Delta_{\infty} u \leq 0$, comparison from below implies $-\Delta_{\infty} u \geq 0$.

We turn now to the proof of

Theorem 3.2 Let $u \in C(U)$. Then $u$ has the $A M L$ property in $U$ if and only if $u$ enjoys comparison with cones in $U$.

Here is a key lemma, for which we recall the notation (2.15) and (2.16).

Lemma 3.3 Let $u \in C(\bar{U})$ enjoy comparison with cones in $U, x_{0} \in U, r<\operatorname{dist}\left(x_{0}, \partial U\right)$. Let $z \in S_{r}\left(x_{0}\right)$ be such than

$$
\frac{u(z)-u\left(x_{0}\right)}{r}=L_{r}^{+}\left(x_{0}\right) .
$$

Then $L_{R}^{+}(z) \geq L_{r}^{+}\left(x_{0}\right)$ for all $0<R \leq \operatorname{dist}(z, U)$. Similarly,

$$
\frac{u(z)-u\left(x_{0}\right)}{r}=L_{r}^{-}\left(x_{0}\right)
$$


implies $L_{R}^{-}(z) \leq L_{r}^{-}\left(x_{0}\right)$ for all $0<R \leq \operatorname{dist}(z, U)$.

Proof. Let $L=L_{r}^{+}(z)=\left(u(z)-u\left(x_{0}\right)\right) / r$ where $\left|z-x_{0}\right|=r$. Translate so that $z=0$. Put $\hat{e}=-x_{0} /\left|x_{0}\right|$ and note that $u(0)=u\left(x_{0}\right)+L\left|x_{0}\right|$. For small $r_{0}>0$ further note that

$$
u\left(-r_{0} \hat{e}\right) \leq u\left(x_{0}\right)+L\left|x_{0}+r_{0} \hat{e}\right|=u(0)-r_{0} L .
$$

We also have

$$
u(y) \leq u(0)+L_{R}^{+}(0)|y| \quad \text { for } \quad|y| \leq R
$$

provided dist $(0, U)<R$. This implies that

$$
\begin{aligned}
u(x) \leq u\left(-r_{0} \hat{e}\right)+ & \max _{y \in S_{R-r_{0}}\left(-r_{0} \hat{e}\right)} \frac{u(y)-u\left(-r_{0} \hat{e}\right)}{R-r_{0}}\left|x+r_{0} \hat{e}\right| \\
& \leq u\left(-r_{0} \hat{e}\right)+\max _{y \in S_{R-r_{0}}\left(-r_{0} \hat{e}\right)}\left(u(0)+L_{R}^{+}(0)|y|-u\left(-r_{0} \hat{e}\right)\right) \frac{\left|x+r_{0} \hat{e}\right|}{R-r_{0}}
\end{aligned}
$$

for $\left|x+r_{0} \hat{e}\right| \leq R-r_{0}$. Put $x=0$ and invoke (3.6) to find

$$
u(0) \leq u(0)-r_{0} L+\left(L_{R}^{+}(0) R+r_{0} L\right) \frac{r_{0}}{R-r_{0}} .
$$

Rearranging, we have

$$
L\left(1-\frac{r_{0}}{R-r_{0}}\right) \leq L_{R}^{+}(0) \frac{R}{R-r_{0}} .
$$

Now send $r_{0} \downarrow 0$ to find that $L=L_{r}^{+}\left(x_{0}\right) \leq L_{R}^{+}(0)$ for every suitable $R$, as claimed.

Proof of Theorem 3.2. Let $u$ enjoy comparison with cones, $u, v \in C(\bar{U}), V \subset \subset U$ be open and connected and $u=v$ on $\partial V$. Assume that $\|D u\|_{L^{\infty}(U)}>\|D v\|_{L^{\infty}(U)}$. Then by Lemma 2.7 there exists $x_{0} \in V$ such that

$$
L^{+}\left(x_{0}\right)>\|D v\|_{L^{\infty}(V)} .
$$

where $L^{+}$refers to $u$. Fix a small $\delta>0$ and define iteratively a doubly infinite sequence $x_{j}$, $j=\ldots,-1,0,1, \ldots$, so that

$$
\left|x_{j}-x_{j-1}\right|=\operatorname{dist}\left(x_{j-1}, \partial V\right)
$$

and

$$
\begin{aligned}
& \frac{u\left(x_{j}\right)-u\left(x_{j-1}\right)}{\left|x_{j}-x_{j-1}\right|}=L_{\left|x_{j}-x_{j-1}\right|}^{+}\left(x_{j-1}\right) \geq L^{+}\left(x_{0}\right) \quad \text { for } \quad j>0, \\
& \frac{u\left(x_{j}\right)-u\left(x_{j-1}\right)}{\left|x_{j}-x_{j-1}\right|}=L_{\left|x_{j}-x_{j-1}\right|}^{+}\left(x_{j}\right) \geq L^{+}\left(x_{0}\right) \quad \text { for } \quad j \leq 0,
\end{aligned}
$$


the last inequalities always holding in view of Lemma 3.3. This construction relies on Lemmas 3.3 and Lemma 2.7 (i). If some $x_{j}$ is in the boundary of $V$, we stop at that point and do not continue to larger indices if $j>0$ nor smaller if $j<0$. This is a simple case, and we assume the sequence is doubly infinite. We conclude that

$$
u\left(x_{j}\right)-u\left(x_{j-1}\right) \geq\left|x_{j}-x_{j-1}\right| L^{+}\left(x_{0}\right)
$$

which expression may be summed to find

$$
u\left(x_{n}\right)-u\left(x_{-n}\right) \geq L^{+}\left(x_{0}\right) \sum_{i=-n}^{n}\left|x_{j}-x_{j-1}\right| .
$$

It follows that there are limit $x_{ \pm n} \rightarrow x_{ \pm \infty} \in \bar{V}$. In fact, $x_{+\infty} \in \partial V$ as $\left|x_{j}-x_{j-1}\right|=$ dist $\left(x_{j-1}, V\right)$ and tends to 0 ; similarly $x_{-\infty} \in \partial V$. Then we have

$$
v\left(x_{\infty}\right)-v\left(x_{-\infty}\right)=u\left(x_{\infty}\right)-u\left(x_{-\infty}\right) \geq L^{+}\left(x_{0}\right) \sum_{j=-\infty}^{\infty}\left|x_{j}-x_{j-1}\right| .
$$

Thus

$$
\begin{aligned}
L^{+}\left(x_{0}\right) \sum_{j=-\infty}^{\infty}\left|x_{j}-x_{j-1}\right| \leq v\left(x_{\infty}\right)-v\left(x_{-\infty}\right) & =\sum_{j=-\infty}^{\infty}\left(v\left(x_{j}\right)-v\left(x_{j-1}\right)\right) \\
& \leq\|D v\|_{L^{\infty}(V)} \sum_{j=-\infty}^{\infty}\left|x_{j}-x_{j-1}\right|,
\end{aligned}
$$

a contradiction to $L^{+}\left(x_{0}\right)>\|D v\|_{L^{\infty}(V)}$.

A corollary of the various equivalences is that merely differentiable solutions of an eikonal equation also solve $-\Delta_{\infty} u=0$.

Corollary 3.4 Let $u$ be everywhere differentiable in $U$ and $|D u(x)|$ be constant in $U$. Then $u$ solves $-\Delta_{\infty} u=0$ in $U$ and has the $A M L$ property.

Proof. Suppose $u \leq a+b\left|x-x_{0}\right|$ on $\partial V \backslash\left\{x_{0}\right\}$ where $V \subset \subset U$ is connected. We may assume $a=0$. If $u-\left(a+b\left|x-x_{0}\right|\right)$ has a positive maximum at some point $\hat{x} \in V \backslash\left\{x_{0}\right\}$ then $b=|D u(x)|$, which is constant. If $b=0$ there is then nothing to prove. If $b \neq 0$, the two functions we wish to compare are both viscosity solutions of the eikonal equation, 
comparable on the boundary, and hence everywhere by a result of Crandall and Lions [8]. The result now follows from Theorem 3.1.

Remark 3.5 It follows that the regularity of solutions of $-\Delta_{\infty} u=0$ can be no better than that of everywhere differentiable solutions of $|D u|=1$. But this does not preclude $C^{1}$; see P. L. Lions [14]. An alternative proof is to note that both $u$ and $v=-u$ are solutions of the eikonal equation in the viscosity sense under the current assumptions, and therefore $u$ and $-u$ are both semiconvex and so $u$ is $C^{1,1}$. Aronsson [3] proved this regularity for $C^{1}$ solutions of the eikonal equation in 2 dimensions for the same purpose. Lions' methods imply the semiconvexity of viscosity solutions.

\section{Linearly Bounded Subsolutions on $\mathbb{R}^{n}$}

Next we prove a Liouville-type theorem:

Theorem 4.1 Let $u \in C\left(\mathbb{R}^{n}\right)$ be a solution of $-\Delta_{\infty} u \leq 0$ in $\mathbb{R}^{n}$. Assume that $a \in \mathbb{R}$, $p \in \mathbb{R}^{n}$ and $u(x) \leq a+\langle p, x\rangle$ for $x \in \mathbb{R}^{n}$. Then $u(x)=u(0)+\langle p, x\rangle$ for $x \in \mathbb{R}^{n}$.

Via rotation and scaling, we may assume that either $p=0$ or $p=e_{1}$ in the assumptions of Theorem 4.1. In the former case, Corollary 2.6 shows that $u$ is constant and we are done. In the latter case, which we now assume, Corollary 2.6 yields

$$
|D u(x)| \leq 1 \text { a.e. }
$$

As once before, we switch to the notation $(x, y) \in \mathbb{R} \times \mathbb{R}^{N-1}$ to denote points of $\mathbb{R}^{N}$. Without loss of generality, we may replace $u$ by $u-a$ and take $a=0$.

Thus let $u(x, y)$ solve $-\Delta_{\infty} u \leq 0$ and satisfy

$$
u(x, y) \leq x
$$

It suffices to prove that $u$ is then linear. The next result is a useful monotonicity property.

Lemma 4.2 Let $-\Delta_{\infty} u \leq 0$ in $\mathbb{R}^{N}$ and (4.2) hold. Then

$$
\delta(x, y)=u(x, y)-x
$$


satisfies

$$
\left\{\begin{array}{l}
x \rightarrow \delta(x, y) \quad \text { is nonincreasing and the limits } \lim _{x \rightarrow-\infty} \delta(x, y) \\
\text { are independent of } y \in \mathbb{R}^{N-1} .
\end{array}\right.
$$

Proof. Since $|D u| \leq 1$ by (4.1), the derivative of $\delta(x, y)=u(x, y)-x$ with respect to $x$ is nonpositive and $x \rightarrow u(x, y)-x$ is nonincreasing (as is $y_{i} \rightarrow u(x, y)-y_{i}$ for $i=1, \ldots, N-1$ ). The relation

$$
(u(x, y)-u(\lambda, 0))^{2}=(x+\delta(x, y)-(\lambda+\delta(\lambda, 0)))^{2} \leq(x-\lambda)^{2}+|y|^{2},
$$

which follows from $|D u| \leq 1$, leads to

$$
2(x-\lambda)(\delta(x, y)-\delta(\lambda, 0))+(\delta(x, y)-\delta(\lambda, 0))^{2} \leq|y|^{2}
$$

Let $\delta(-\infty, y)$ denote the $\operatorname{limit}_{x \rightarrow \infty} \delta(x, y)$. Putting $x=2 \lambda$ in (4.5), dividing by $\lambda$ and sending $\lambda \rightarrow-\infty$ yields $\delta(-\infty, y)-\delta(-\infty, 0) \geq 0$. Putting $\lambda=2 x$ and sending $x \rightarrow-\infty$ yields the opposite inequality, and we conclude that $\delta(-\infty, y)=\delta(-\infty, 0)$ for all $y \in \mathbb{R}^{N-1}$.

Remark 4.3 As $\delta(x, y) \leq 0$ increases as $x$ decreases, $\delta(-\infty, y)$ is finite. The limit $\delta(\infty, y)$ is also independent of $y$ by similar arguments. These arguments, depending only on the bound $|D u| \leq 1$, do not show that $\delta(\infty, y)$ is finite.

Proof of Theorem 4.1. We may now assume that $u(x, y)=x+\delta(x, y) \leq x+\delta(-\infty, 0)$, replace $u$ by $u-\delta(-\infty, 0)$ and assume that $u \leq x$ and $x-u(x, y) \rightarrow 0$ monotonically as $x \rightarrow-\infty$. In particular,

$$
\delta(-\infty, 0)=0
$$

If $x_{0}-u\left(x_{0}, y_{0}\right)>0$ holds, then $v(x, y)=u\left(x+x_{0}, y\right)-x_{0}$ has all the desired properties and $v(0,0)<0$, so we may simply assume

$$
u(0,0) \leq-\kappa<0 .
$$

If we contradict $(4.6),(4.7)$, then we conclude that $u(x, y)=x$, whence the result. 
Recall $|D u| \leq 1$. Combining this with (4.7), we have

$$
u(x, y) \leq-\kappa+|(x, y)|
$$

To proceed, we construct a cone with vertex $(-r, 0)$ to the left of the origin with a slope a bit less than 1 , and which majorizes $u$ on the sphere

$$
S_{R}=\{(x, y):|(x, y)-(-r, 0)|=R\}
$$

and at $(-r, 0)$, and therefore on $\{(x, y):|(x, y)-(-r, 0)| \leq R\}$. Upon letting $R=2 r \rightarrow \infty$ we will find that $\delta(-\infty, 0)<0$, a contradiction to (4.6). Thus $u(x, y)=x$, the desired conclusion.

For the construction, we first use the relations

$$
u \leq x \quad \text { and } \quad u \leq-\kappa+|(x, y)|
$$

to estimate $\max _{S_{R}} u$ where $r<R$. To do this, we examine what we would do to prove $u \leq M$ on $S_{R}$. If $x \leq M$, then $u(x, y) \leq M$, so we only need to consider $M \leq x$. To estimate $u$ in this case, we compute

$$
\max _{S_{R} \cap\{M \leq x\}}(-\kappa+|(x, y)|)=-\kappa+\max _{S_{R} \cap\{M \leq x\}}|(x, y)| .
$$

Now on $S_{R} \cap\{M \leq x\}$ we have

$$
\begin{aligned}
|(x, y)|^{2}=x^{2}+y^{2} & =(x+r)^{2}+y^{2}-2 r x-r^{2} \\
& =R^{2}-2 r x-r^{2} \\
& \leq R^{2}-2 r M-r^{2}
\end{aligned}
$$

so we have $-\kappa+|(x, y)| \leq M$ on $S_{R} \cap\{M \leq x\}$ provided that

$$
-\kappa+\sqrt{R^{2}-2 r M-r^{2}} \leq M
$$

clearly we are only concerned with $M \leq R-r$. This latter condition is equivalent to

$$
M \leq-\kappa-r+\sqrt{R^{2}+2 \kappa r} .
$$

We conclude that (4.9) implies

$$
\max _{S_{R}} u \leq-\kappa-r+\sqrt{R^{2}+2 \kappa r}
$$


It follows now by comparison with cones that

$$
u(x, y) \leq C(x, y)=-r+\frac{-\kappa+\sqrt{R^{2}+2 \kappa r}}{R}|(x, y)-(-r, 0)|
$$

for $|(x, y)-(-r, 0)| \leq R$, since the inequality holds at the vertex $(-r, 0)$ and on the boundary $S_{R}$. To conclude, we put $x=-s$ where $0 \leq s<r, y=0$, to find

$$
u(-s, 0)-(-s) \leq\left(-1+\frac{-\kappa+\sqrt{R^{2}+2 \kappa r}}{R}\right)(r-s) .
$$

We then put $R=2 r$ and let $r \rightarrow \infty$ to deduce

$$
u(-s, 0)-(-s) \leq-\frac{\kappa}{4} \quad \text { and hence } \quad \delta(-\infty, 0) \leq-\frac{\kappa}{4}
$$

which contradicts $\delta(-\infty, 0)=0$.

Corollary 4.4 Let $u$ be a solution of $-\Delta_{\infty} u \leq 0$ in $U$ and $\hat{x} \in U$. Assume there exists $p \in \mathbb{R}^{n}$ such that

$$
u(x) \leq u(\hat{x})+\langle p, x-\hat{x}\rangle+\mathrm{o}(|x-\hat{x}|)
$$

Then $u$ is differentiable at $\hat{x}$ and $D u(\hat{x})=p$.

Proof. By Lemma $2.5 u$ is Lipschitz continuous near $\hat{x}$, let $L$ be a Lipschitz constant for $u$ in the ball $B_{r}(\hat{x})$. Consider the function

$$
v_{\lambda}(x)=\frac{u(\lambda x+\hat{x})-u(\hat{x})}{\lambda}
$$

where $\lambda>0$; it is bounded and Lipschitz with constant $L$ on the ball $B_{r / \lambda}$. If $D u(\hat{x}) \neq p$, then there exists $x_{j}$ such that $0<\left|x_{j}\right| \rightarrow 0$ and

$$
\liminf _{j \rightarrow \infty} \frac{\left|u\left(x_{j}+\hat{x}\right)-\left\langle p, x_{j}\right\rangle-u(\hat{x})\right|}{\left|x_{j}\right|}>0 .
$$

Put $\lambda_{j}=\left|x_{j}\right|$ and select a locally uniformly convergent subsequence of the functions $v_{\lambda_{j}}$ whose limit we denote by $v$. By viscosity solution theory, $-\Delta_{\infty} v \leq 0$. By $(4.11), v(x) \leq$ $\langle p, x\rangle$. From Theorem 4.1 and $v(0)=0, v(x)=\langle p, x\rangle$. Finally, if $x_{j} /\left|x_{j}\right| \rightarrow x,(4.12)$ yields $|v(x)-\langle p, x\rangle|>0$, a contradiction. 


\section{References}

[1] G. Aronsson, Extension of functions satisfying Lipschitz conditions, Arkiv für Mate. 6 (1967), 551-561.

[2] G. Aronsson, On the partial differential equation $u_{x}^{2} u_{x x}+2 u_{x} u_{y} u_{x y}+u_{y}^{2} u_{y y}=0$, Arkiv für Mate. 7 (1968), 395-425

[3] G. Aronsson, On certain singular solutions of the partial differential equation $u_{x}^{2} u_{x x}+$ $2 u_{x} u_{y} u_{x y}+u_{y}^{2} u_{y y}=0$, Manuscripta Math. 47 (1984), 133-151

[4] E. N. Barron, Viscosity solutions and analysis in $L^{\infty}$, in Nonlinear Analysis, Differential Equations and Control (ed. by Clarke and Stern), Kluwer Academic Publishers, 1999.

[5] T. Battharchaya, E. Di Benedetto and J. Manfredi, Limits as $p \rightarrow \infty$ of $\Delta_{p} u_{p}=f$ and related extremal problems, Rend. Sem. Mat. Unvers. Politecn. Torino, Fasciocolo Speciale (1989), Nonlinear PDE's, 15-68.

[6] V. Caselles, J.-M. Morel and C. Sbert, An axiomatic approach to image interpolation,, IEEE Trans. Image Proc., 7 (1996), 376-386.

[7] M. G. Crandall, H. Ishii, and P.-L. Lions, User's guide to viscosity solutions of secondorder partial differential equations, Bull. Am. Math. Soc. 27 (1992), 1-67.

[8] M. G. Crandall and P.-L. Lions, Viscosity Solutions of Hamilton-Jacobi equations, Trans. Amer. Math. Soc. 277 (1983), 1-42.

[9] L. C. Evans, Estimates for smooth absolutely minimizing Lipschitz extensions, Elec. J. Diff. Eq. 1993 (1993), 1-9.

[10] R. Jensen, Uniqueness of Lipschitz extensions minimizing the sup-norm of the gradient, Arch. Rat. Mech. Anal., 123 (1993), 51-74.

[11] P. Juutinen, Minimization problems for Lipschitz functions via viscosity solutions, Annales Academiae Scientiarum Fennicae, Mathematica, Dissertationes 115, 1998.

[12] P. Lindqvist and J. Manfredi, The Harnack inequality for $\infty$-harmonic functions, Elec. J. Diff. Eq. 1995 (1995), 1-5.

[13] P. Lindqvist and J. Manfredi, Note on $\infty$-superharmonic functions, Rev. Mat. Univ. Complut. Madrid 10 (1997) 
[14] P. L. Lions, Generalized Solutions of Hamilton-Jacobi Equations, Research Notes in Mathematics 69, Pitman, Boston, 1982.

[15] Y. Wu, Absolute minimizers in Finsler metrics, Ph.D. dissertation, UC Berkeley, 1995 